\title{
Experimental Investigation of the Structure and Dynamics of Laminar Separation Bubbles
}

\author{
S.J.Chetan ${ }^{1}$ and Hermann Fasel $^{2}$
}

Department of Aerospace and Mechanical Engineering, University of Arizona, Tucson, Arizona, 85721

This work is an experimental investigation of the dynamics of the laminar separation bubbles, which are typically present on the suction side of lifting surfaces at a large angle of attack. The separation bubble was generated on a flat plate by an adverse pressure gradient induced by The adverse pressure gradient was generated by using an inverted wing with a NACA 64 ${ }_{3}-618$ airfoil mounted above the flat plate. Using Particle Image Velocimetry (PIV), a parametric study of the effect of the upstream flow velocity and the induced pressure gradient on the mean flow topology and the unsteady behavior of the separation bubble was carried out in the low-speed water tunnel of the Hydrodynamics Laboratory at the University of Arizona. The topology of the laminar separation bubble, and in particular the unsteady flow dynamics, were found to be strongly dependent on these parameters. For certain conditions, strong vortex shedding near the reattachment region of the bubble was observed, which is a characterisc behavior of short bubbles. High-resolution spatio-temporal PIV measurements were made to analyze the formation and breakdown of these flow structures. The frequency of vortex shedding was determined from Fourier analysis of the time series of the velocity fluctuations. The non-dimensionalised frequencies were found to be nearly independent of the Reynolds number for the range of Reynolds numbers investigated here.

\section{Nomenclature}

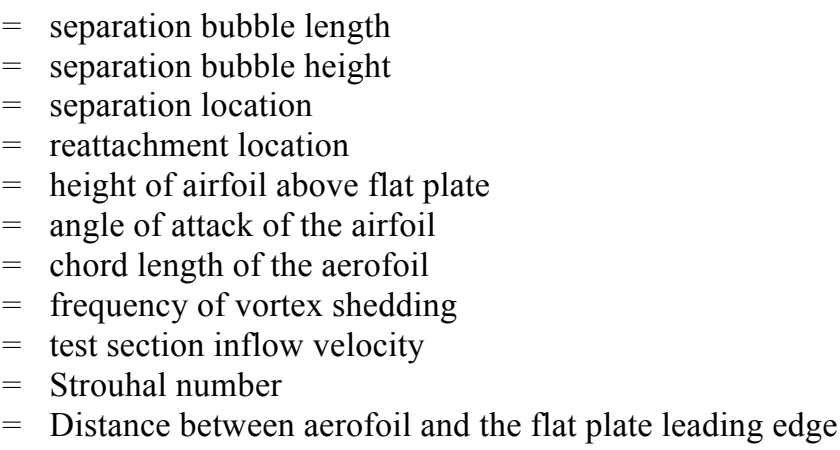

\section{Introduction}

A laminar boundary layer forming on a continuous smooth surface separates from the surface when it encounters a strong enough adverse pressure gradient. Usually the separated shear layer is hydrodynamically unstable, resulting in laminar-turbulent transition that starts at a short distance downstream of the point of separation. The process of transition is initiated by the amplification of small disturbances in the unstable laminar layer. After transitioning, the flow eventually reattaches to the surface due to turbulent mixing, and thus forming a closed region of separated flow, the so-called laminar separation bubble. An instantaneous flow visualization (snapshot) of a typical separation bubble observed in our investigations is shown in Fig. 1.

\footnotetext{
${ }^{1}$ Postdoctoral Research Associate.

${ }^{2}$ Professor, AIAA Member.
} 


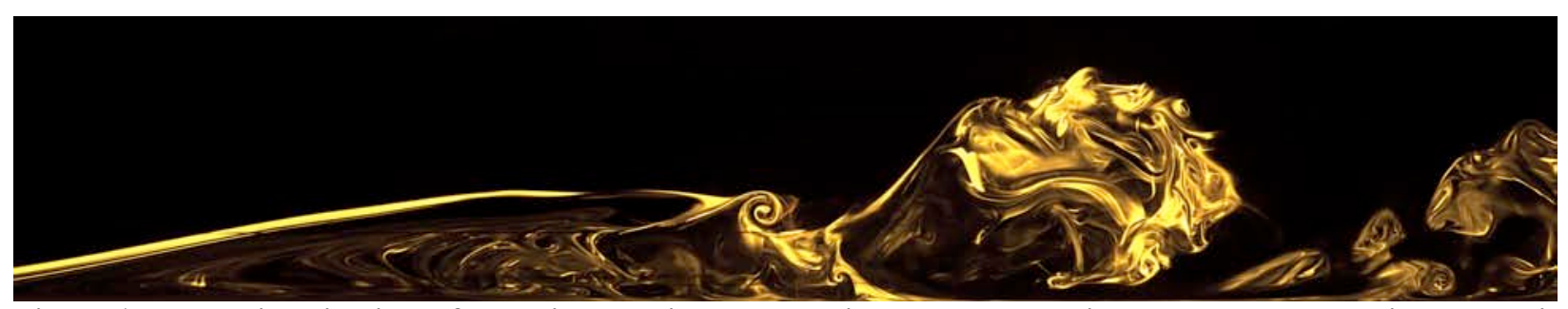

Figure 1. Flow visualization of a typical laminar separation bubble showing the vortex shedding. Flow is from left to right.

A schematic of a time-averaged laminar separation bubble, as described by Mayle ${ }^{2}$ and by Horton ${ }^{7}$ is shown in Fig.2. Downstream of the point of separation, $X s$, the fluid velocity near the wall is significantly less than the velocity of the flow outside the separation bubble. This region is referred to as the 'dead air' region, where the surface pressure is almost constant, as indicated in the pressure distribution curve. The reattachment occurs at a point where the value of pressure on the wall is nearly equal to that which would have existed had there been an attached boundary layer with no separation bubble present.

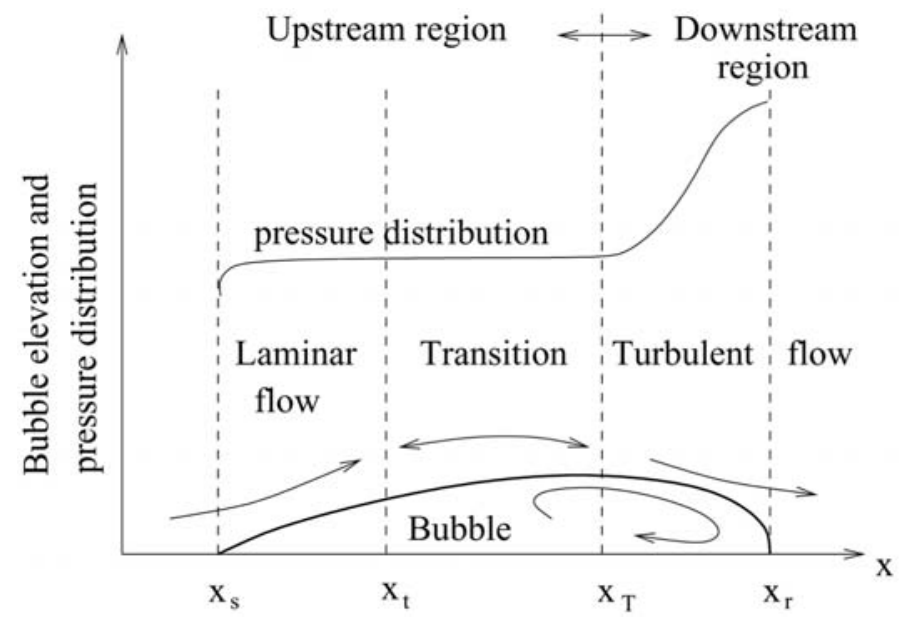

Figure 2. Section view of a laminar separation bubble (from Mayle ${ }^{2}$ )

Boundary layer separation occurs in many practical flow situations such as Micro Air Vehicles (MAV), Unmanned Aerial Vehicles (UAV), leading edges of thin airfoils and turbomachinery blades, or whenever the Reynolds numbers involved are relatively low. Flow separation in practical applications can lead to massive degradation of mean aircraft stability and aerodynamic performance characteristics (McMichael and Francis ${ }^{1}$ ). The degradation of performance is seen to be due to a dramatic change in pressure drag. Also, the unstable nature of the separation bubble leads to transition and the formation of a boundary layer that is turbulent at low Reynolds numbers. Thus the separation bubbles in these cases constitute a laminar separation and the eventual transition to turbulence, at any given time (Häggmark et $a l^{8}$ ). A better understanding of the fundamental physics of laminar separation bubbles is essential for the development of successful and efficient separation control strategies (Gad-el$\mathrm{Hak}^{9}$ ), which ultimately would lead to improved aerodynamic performance of flight vehicles.

The laminar separation bubbles have received a great deal of attention over the years. Jones ${ }^{10}$, working on thin aerofoils for high-speed flight, was one of the first to report experimental results involving laminar separation bubbles. The experimental work of Gaster ${ }^{4}$ was conducive in the general understanding of the bubble structure. He also introduced a pressure-parameter based criterion for the classification of laminar separation bubbles. Owen \& Klanfer $^{11}$ were the first to classify laminar separation bubbles as either 'short' or 'long'. This classification was based on the length of the bubble and the displacement thickness at the point of separation. Based on the Reynolds number and the shape of the aerofoil, the short bubbles are seen to have a minor effect on the pressure gradient. With an increase in the angle of attack, the 'short' bubble is seen to break open or 'burst' into a 'long' bubble. Van den Berg ${ }^{12}$ showed that for a NACA $64_{1}-012$ airfoil, the bubble bursting causes the bubble reattachment point to

2

American Institute of Aeronautics and Astronautics 
move downstream towards the trailing edge so that the overall pressure distribution around the aerofoil - and therefore the circulation - is profoundly (adversely) changed.

The bursting criterion proposed by Gaster relates the momentum-thickness Reynolds number at the location of separation (when the bubble is not present), $\operatorname{Re} \theta_{s}$ and a non-dimensional pressure gradient parameter $P$.

$$
P=\frac{\theta_{s}^{2}}{v} \frac{\Delta U}{\Delta x}
$$

In the experiments, tripping of the boundary layer provided the measurement of the inviscid pressure distribution and the velocity difference, $\Delta U$, over the length of the bubble.

The experimental setup used in the present work is similar to the one used by Gaster, where in an inverted aerofoil-shaped high-aspect ratio displacement body was used to generate the required adverse pressure gradient to form a separation bubble on a flat plate. Diwan et $a l^{13}$ proposed a refined bursting criterion, which takes into account not only the length of the separation bubble but also the maximum height, as it was argued that the transition to turbulence was initiated roughly at a streamwise location corresponding to the maximum height. Their refined nondimensional pressure gradient parameter is given as

$$
P_{\text {refined }}=\frac{h^{2}}{v} \frac{\Delta U}{\Delta x}
$$

where $\Delta U / \Delta x$ is the actual mean velocity gradient across the bubble. The relation between the Reynolds number at the maximum height of the bubble $\operatorname{Re}_{h}$ and the refined pressure parameter $P_{\text {refined }}$ was seen to clearly distinguish between 'short' and 'long' bubbles, thereby resulting in a single parameter criterion that indicates the transition between them. Marxen \& Henningson ${ }^{14}$ suggest that there are at least two forms of bubble bursting, depending on the characteristics of the flow at reattachment, i.e., laminar, turbulent reattachment.

Two-dimensional computational studies carried out by Pauley, Moin \& Reynolds ${ }^{15}$, Ripley \& Pauley ${ }^{16}$, Lin $\&$ Pauley ${ }^{17}$, Alam \& Sandham ${ }^{18}$ provide some useful understanding of the flow physics of laminar separation bubbles. The results of the simulations show a reasonable agreement with experimental data available in the literature. It is observed from the numerical simulations that the separation bubble is often unsteady, with vortex shedding setting in at higher values of adverse pressure gradients. It is also noted that incoming disturbances have a significant effect on the stability of the separation bubble, leading to unsteadiness. Pauley, Moin \& Reynolds ${ }^{15}$ suggest that bubble bursting correlates to the demise of vortex shedding, thereby leading to a fully steady, long laminar separation bubble, and that bubbles with high levels of reverse flow (long bubbles) appear to be absolutely unstable.

Hammond \& Redekopp ${ }^{19}$ performed theoretical and numerical studies of separation bubbles with respect to local and global instabilities based on the Orr-Sommerfeld equation. Velocity profiles consisting of Falkner-Skan profiles with reverse flow and an additional term containing two perturbation parameters, which enabled a larger and independent variation in the amount and extent of the reverse flow, were studied. They reported that the amount of maximum reverse-flow velocity was a deciding factor as to whether an inflection point instability mode leads to an absolutely unstable behavior or not. Hammond \& Redekopp ${ }^{19}$ also suggested a possible connection with the onset of large-scale vortex shedding as observed in Pauley et al. (1990) when the strength of the applied adverse pressure gradient was increased in their simulations.

The present experiments are directly linked to the Direct Numerical Simulations, which are carried in our Computational Fluid Dynamics Laboratory at the University of Arizona (Hosseinverdi and Fasel ${ }^{23}$ ). The ultimate goal of this research is to make major contributions towards the understanding of the fundamental physics of separation bubbles and in particular, in the understanding of the intricate interaction of transition and separation, and the role of this interaction in the phenomenon of bubble bursting.

\section{Experimental Setup}

The experiments were conducted in the $4.5 \mathrm{~m} \times 1.5 \mathrm{~m} \times 0.45 \mathrm{~m}$ test-section of the open surface water tunnel in the Hydrodynamics Laboratory of the University of Arizona. The turbulence intensity, $T_{i}$ was measured to be less than $0.1 \%$ at a flow velocity $U_{\infty}=0.05 \mathrm{~m} / \mathrm{s}$. The separation bubble was generated on a flat plate, having an elliptical leading edge, mounted in the test section at a height of $0.1 \mathrm{~m}$ from the bottom wall. The adverse pressure gradient was generated by using an inverted wing with a NACA $64_{3}-618$ airfoil section mounted above the flat plate, the span and chord of which were $1.2 \mathrm{~m}$ and $0.3 \mathrm{~m}$, respectively. Suction was provided on the wing, so as to prevent flow 
separation at high angles of attack. Suction holes having a diameter of $0.001 \mathrm{~m}$ were drilled on the suction side of the wing spanning from chord length of $50 \%$ to $90 \%$, over the entire span. The pressure head necessary for suction was imparted by locating the reservoir below the water level of the tunnel, thus using gravity to guarantee steady suction. The volume flow rate can be adjusted by means of adjusting the vertical height of the suction exit. The schematic of the experimental arrangement is given in figure 3 .

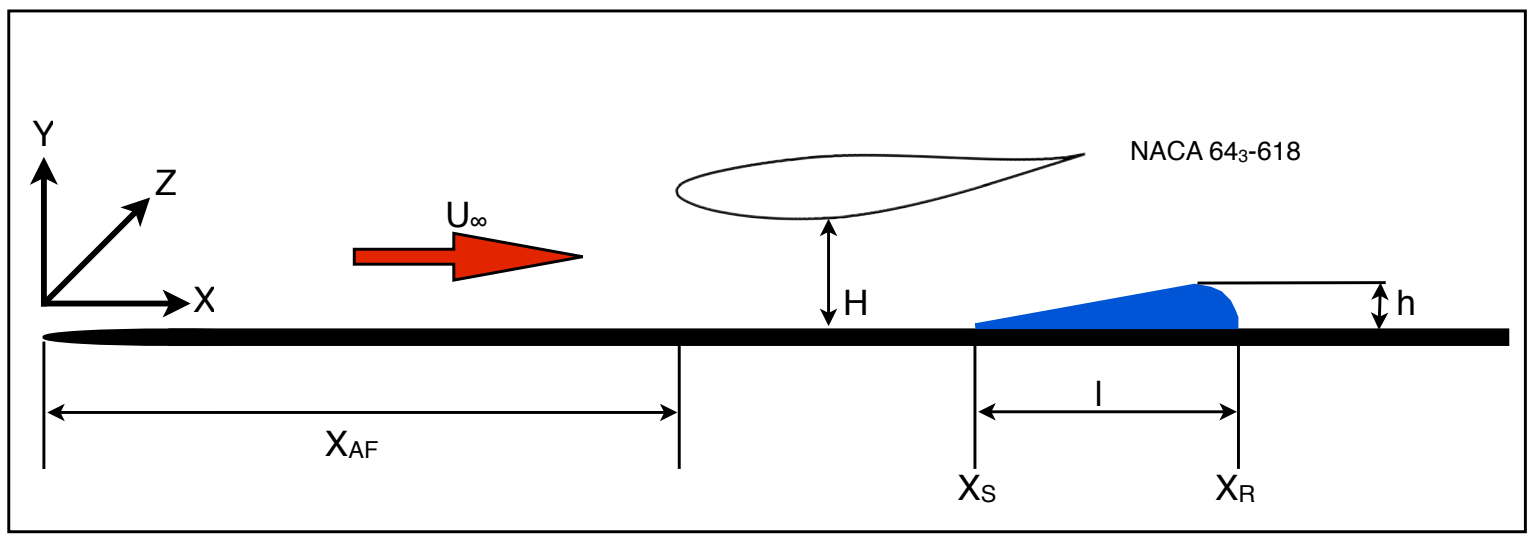

Figure 3. Experimental arrangement showing the position of the inverted wing with respect to the flat plate.

A Particle Image Velocimetry (PIV) system was used to measure the vertical and horizontal components of velocity in the midspan plane of the tunnel test-section. The PIV system by LaVision, consisted of a double-pulsed $532 \mathrm{~nm} \mathrm{Nd}$ :YAG laser having an output of $120 \mathrm{~mJ} /$ pulse. The light-sheet optics provided a laser sheet having a thickness of approximately $0.002 \mathrm{~m}$ which was parallel to the flow. The laser sheet was introduced into the field of measurement from the downstream part of the test-section by using a mirror aligned at 45 degrees to the direction of flow. This allowed for the capturing of the dynamics of the entire separation bubble in real time. All measurements were made at the centerline of the test section. Two CCD cameras having a resolution of $1600 \times 1200$ pixels were used in parallel (side by side) to capture the flow field of the entire bubble. The laser and the cameras were mounted on a traverse system, which allowed for streamwise movement of the whole system without the need for recalibration. The laser and the CCD cameras were connected to a workstation via a synchronizer, which controlled the timing of the laser and the image acquisition/processing. Hollow glass spheres (Sphericel 110p8 with a specific gravity of $1.1 \mathrm{~g} / \mathrm{cm}^{3}$ ) mixed in water were used as seeding material. The seeding particles had an average diameter of $10 \mu \mathrm{m}$ and appeared in images with diameter of between 3 and 5 pixels. Velocity vectors were derived from double frame pixel PIV images using an adaptive multi-pass cross-correlation algorithm starting with 64 X 64 pixels interrogation windows with window shifting and deformation, leading to $16 \mathrm{X} 16$ pixel interrogation windows with $50 \%$ overlap. This methodology was found to increase the peak value of correlation in comparison to the noise, which resulted in the reduction of the number of erroneous vectors and the need for vector interpolation to rectify them $\left(\right.$ LaVision $\left.^{6}\right)$.

Flow visualizations with laser-induced fluorescent dye was used to ascertain the global structure of the laminar separation bubbles. A solution of $2 \%$ Rhodamine-B, a nontoxic pigment, was introduced into the flow $1 \mathrm{~m}$ upstream of the displacement body by means of a dye-tube. The dye-tube was enclosed within a low-profile fairing to reduce the effect of its wake. Rhodamine-B, when excited with an appropriate light source, the PIV laser in the present work, fluoresces $\left(\right.$ Merzkirch ${ }^{5}$ ) and this scattered fluorescent light was captured by means of a digital camera fitted with an appropriate narrowband optical notch filter to selectively block the laser light, being emitted at a wavelength of 532nm. Since it was made sure that there was no ambient light in the vicinity of the experimental setup, only the scattered fluorescent light was captured by the camera. Figure.1 shows a typical example of the flow visualization images obtained using the above-mentioned technique. 


\section{Results}

Experiments were conducted at a range of upstream flow velocities $\left(0.027 \mathrm{~m} / \mathrm{s} \leq U_{\infty} \leq 0.087 \mathrm{~m} / \mathrm{s}\right)$ and two different angles of attack of the displacement body. Changing the angle of attack of the displacement body varied the intensity of the imposed pressure gradient. Due to the complex nature of a number of phenomena investigated during this experimental program, the following discussion of results will highlight some the important observations in this continuing research program. Results for three different upstream velocities, which represent low, medium and high velocities in the aforementioned range, will be presented in the following sections. The leading edge of the wing was positioned at a location $X_{A F}=0.58 \mathrm{~m}$ downstream of the flat plate leading edge, and at a height $H=0.12 \mathrm{~m}$ above the flat plate surface (see Fig 3). Data were acquired for a total of $300 s$ with sufficient time allowed for the flow to settle between each experimental run involving a change in flow velocity and/or angle of attack.

Fig.4 shows the time-averaged flow field and representative velocity profiles upstream of the aerofoil for a freestream velocity of $U_{\infty}=0.027 \mathrm{~m} / \mathrm{s}$. The flow accelerates as it approaches the aerofoil and the effect of this is seen in the mean streamline pattern and the velocity profiles. The observed acceleration of flow is due to the presence of the wing.

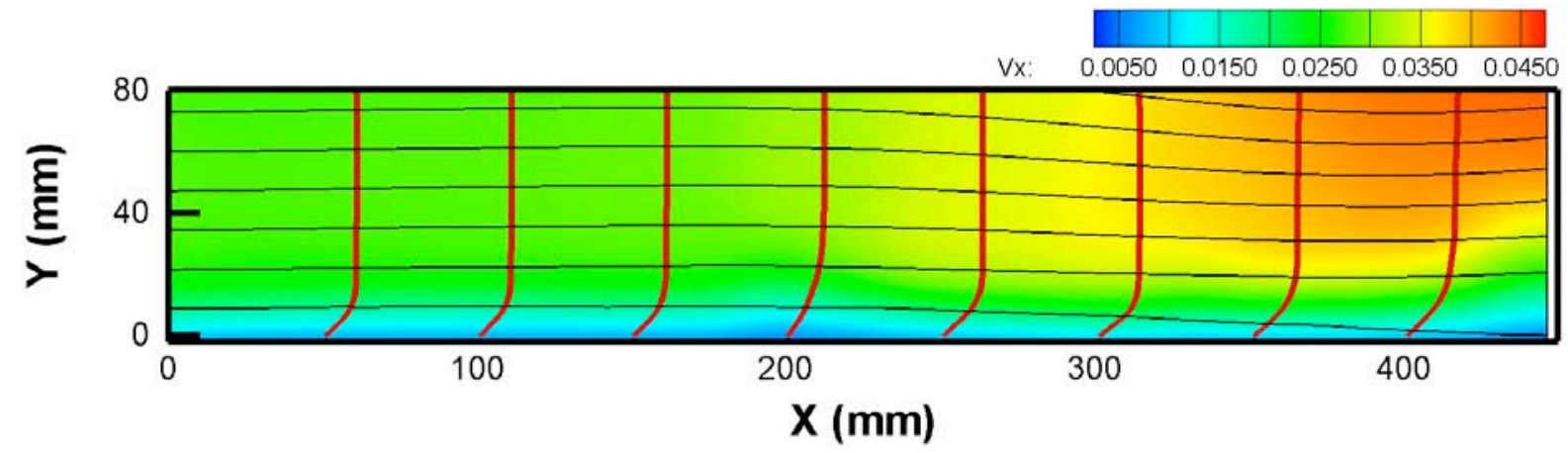

Figure 4. Time-averaged flow field upstream of the aerofoil. Also shown are the mean velocity profiles. The leading edge of the aerofoil is approximately at the location $x=375 \mathrm{~mm}$ in the scales shown in the figure. Freestream velocity $U_{\infty}=0.027 \mathrm{~m} / \mathrm{s}$.

\section{A. Geometry of the laminar separation bubble}

The laminar separation bubble forms on the flat plate due to the adverse pressure gradient imposed by the inverted wing. The instantaneous PIV data was time-averaged over 1500 image pairs Figure 5 shows the timeaveraged structure of the laminar separation bubble, along with the velocity profiles within. The flow separates at the location $X_{S}=0 \mathrm{~mm}$ and at this point the dividing streamline departs gradually from the wall, with the shear layer returning to the wall at the reattachment point $X_{R}=450 \mathrm{~mm}$. A strong counter-rotating vortex is observed within the separation bubble near the point of reattachment. The length of separation, $l$, is considered as the distance between the points of separation and reattachment of the time-averaged separation bubble. The effect of upstream flow velocity $U_{\infty}$ on the length and height is shown in figure 6 and figure 7.

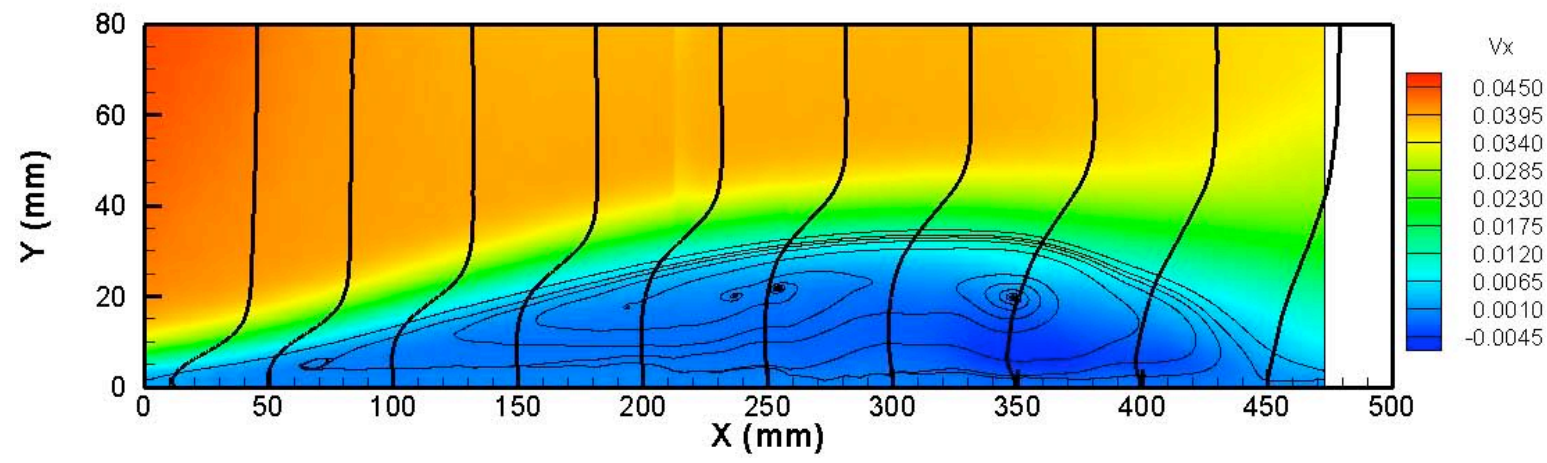

Figure 5. Time-averaged flow field upstream of the aerofoil. Also shown are the mean velocity profiles. The leading edge of the aerofoil is approximately at the location $X=375 \mathrm{~mm}$ in the scales shown in the figure. Freestream velocity, $U_{\infty}=0.027 \mathrm{~m} / \mathrm{s}$. 
The length of the separation bubble is seen to decrease with increasing flow velocities, with the reattachment point moving upstream. The separation location also remains the same, which is consistent with the findings reported in the literature. The height of the separation bubble decreases as the freestream velocity is increased. In the second set of experiments, the imposed pressure gradient was increased by increasing the angle of attack of the wing. The mean velocity contours of the separation bubble for three different upstream velocities and two different imposed pressure gradients are given in figs 6 and 7, respectively. In the first set, the wing was at an angle of attack of 0 degrees and the in the second set the angle of attack was 5 degrees, which resulted in a stronger pressure gradient imposed on the boundary layer. The location of separation point seems to be independent of the flow velocity, but depends on the strength of the imposed pressure gradient. Figure 8 shows the length and height of the measured separation bubbles and the effect of the change of the imposed pressure gradient. We observed that the length of the bubbles varies approximately linearly with the change in velocity for both sets of experiments. Bubble bursting, as defined by Gaster ${ }^{4}$ would involve a sudden change or jump in the length of the separartion bubble at a critical flow condition brought about by the change in inflow velocity or the imposed pressure gradient. No such sudden departures were observed in the cases presented. This would lead us to believe that the separation bubbles studied in the present work were of the 'short' kind. Further reduction of the inflow velocity combined with a reduction of the imposed pressure gradient could possibly lead towards flow conditions amenable for the bursting of short bubbles.
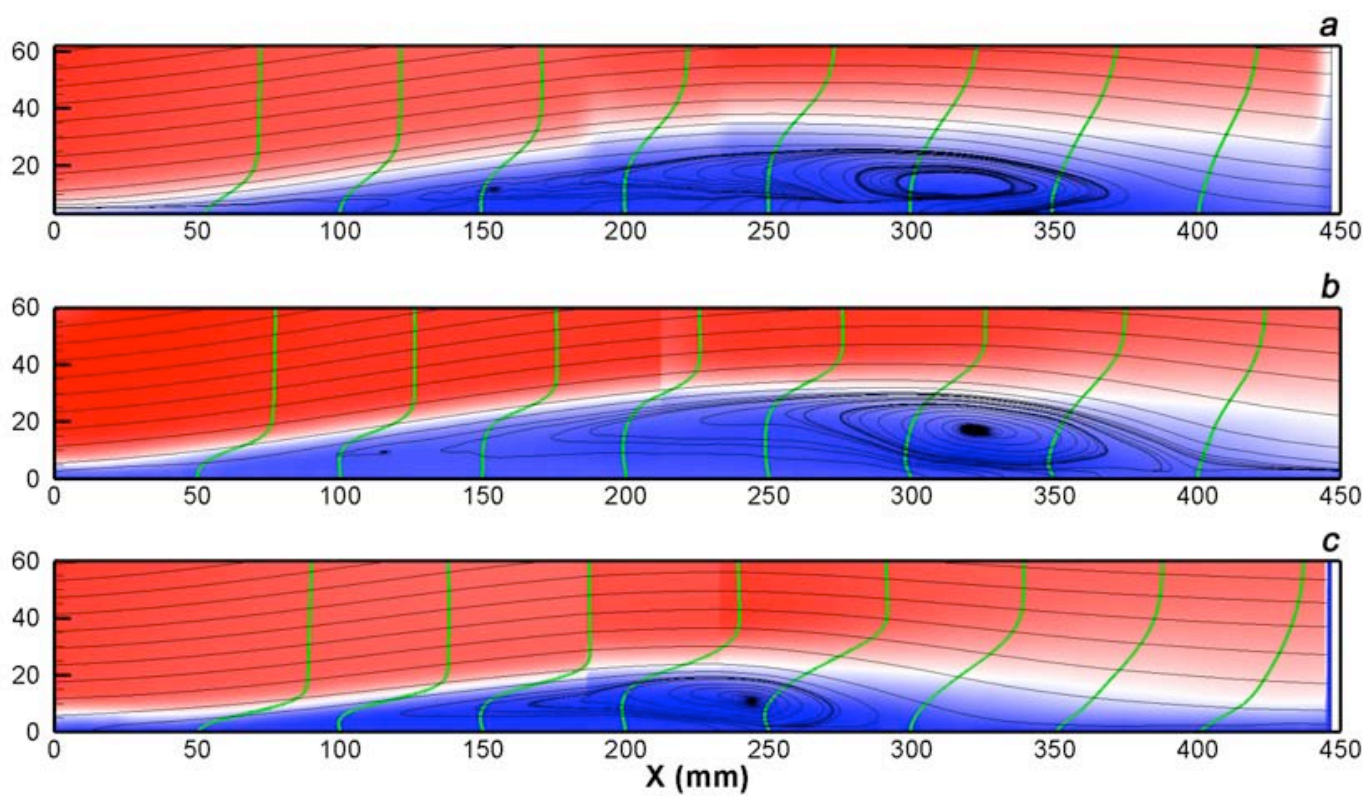

Figure 6. Time-averaged streamlines and velocity profiles of separation bubbles at three different inflow velocities, at a given angle of attack of the aerofoil. Angle of attack of the aerofoil $=0$ degrees $=0.027 \mathrm{~m} / \mathrm{s}(\mathrm{b}) U_{\infty}$ $=0.052 s(c) U_{\infty}=0.087 s$. 

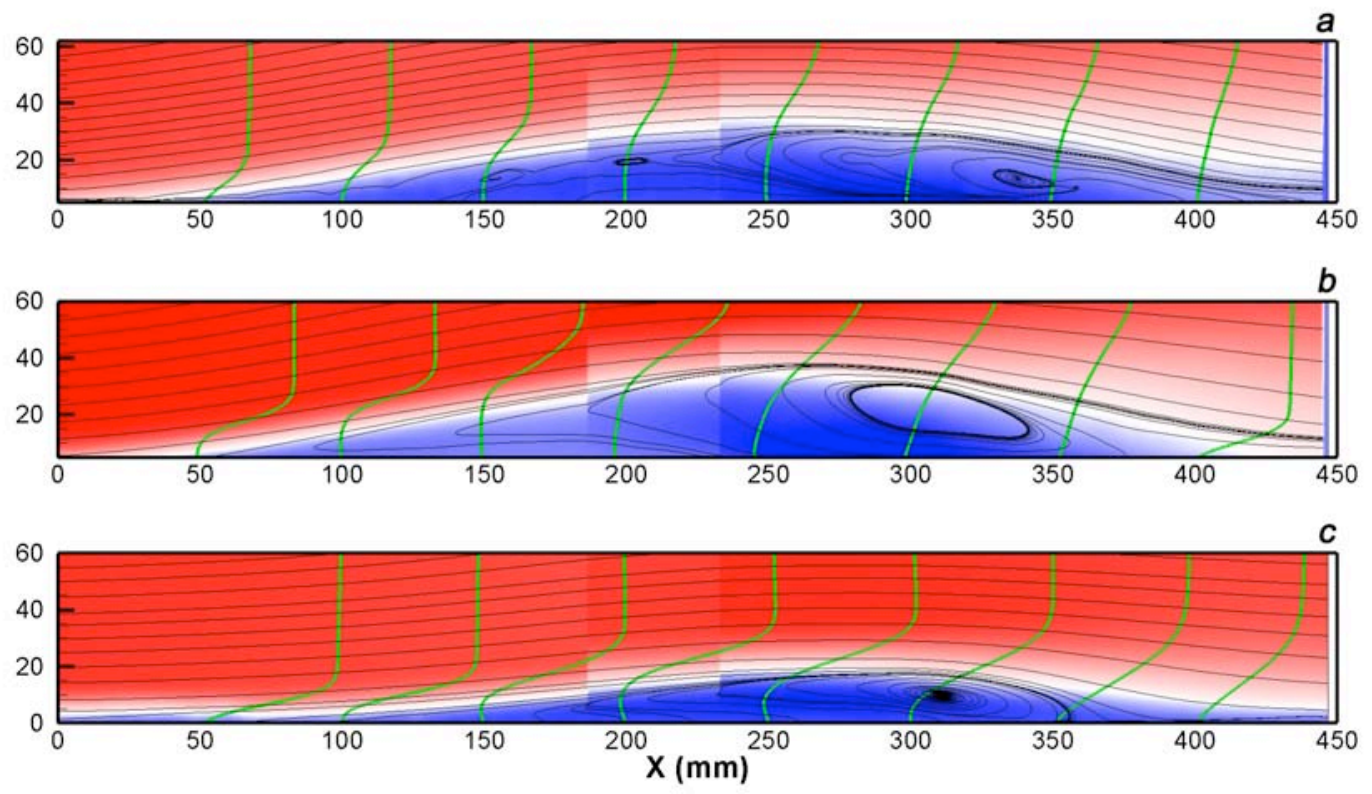

Figure 7. Time-averaged streamlines and velocity profiles of separation bubbles at three different inflow velocities, at a given angle of attack of the aerofoil. Angle of attack of the aerofoil $=5$ degrees. (a) $U_{\infty}=0.027 \mathrm{~m} / \mathrm{s}$ (b) $U_{\infty}=0.052 s$ (c) $U_{\infty}=0.087 \mathrm{~s}$.

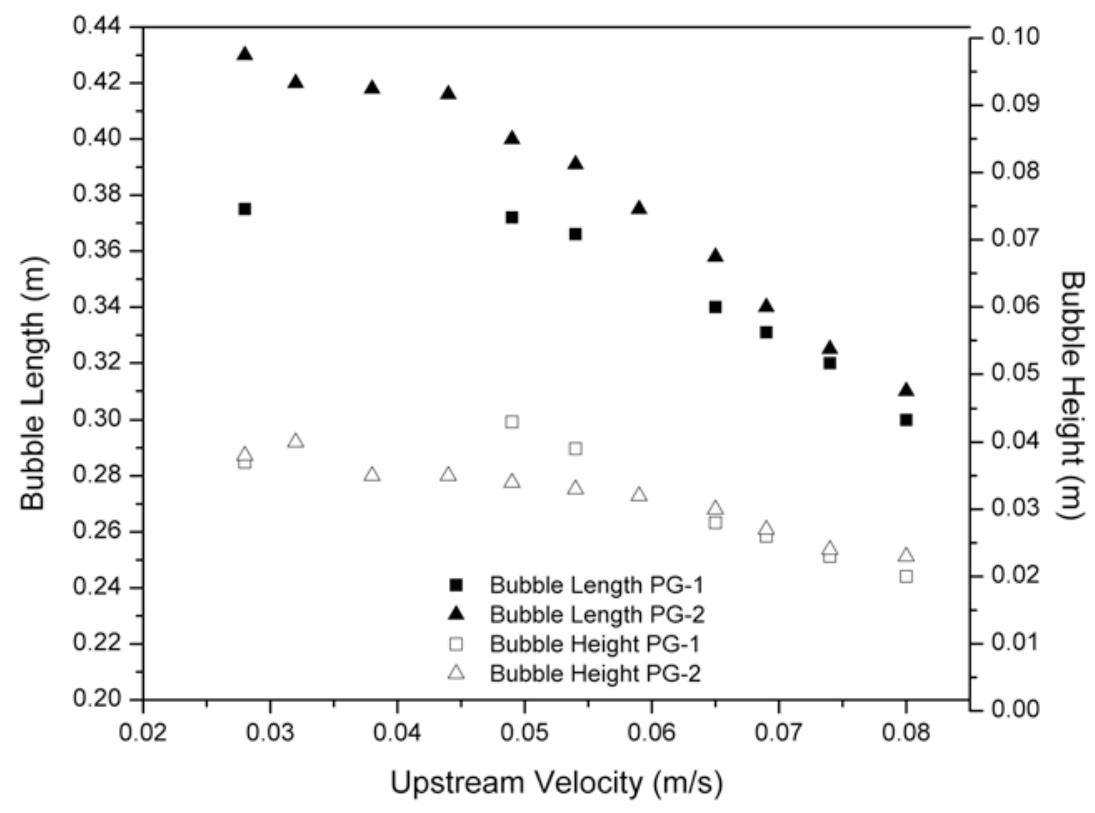

Figure 8 Comparison of the length $I$ and height $h$ of the separation bubbles at two different pressure gradient settings. PG-1 refers to the aerofoil angle of attack of 0 degrees. PG-2 refers to the aerofoil angle of attack of 5 degrees.

\section{B. Unsteady flow development}

The separation bubbles in the present work exhibited an unsteady behavior. The temporal variation of the measured velocity field at different inflow velocities will be presented in this section. Flow visualisation was conducted to better understand the overall structure of the unsteady nature of the separation bubbles. Figure 9 shows snapshots of the flow fields and the associated vortex shedding for the three different inflow velocities. The flow 
visualization provides a useful qualitative overview of the structure of the separation bubble and the highly unsteady nature of the reattachment process.

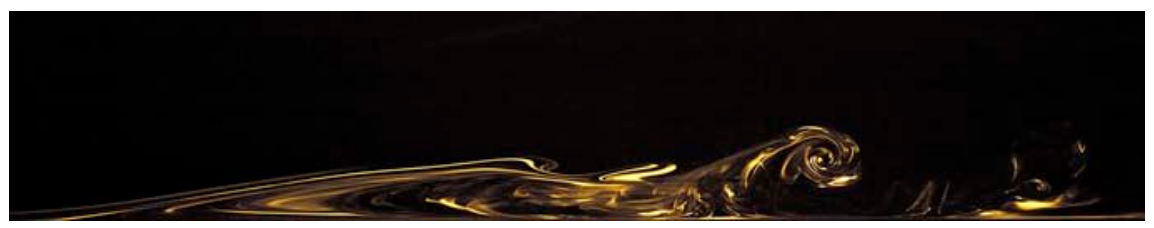

(a)

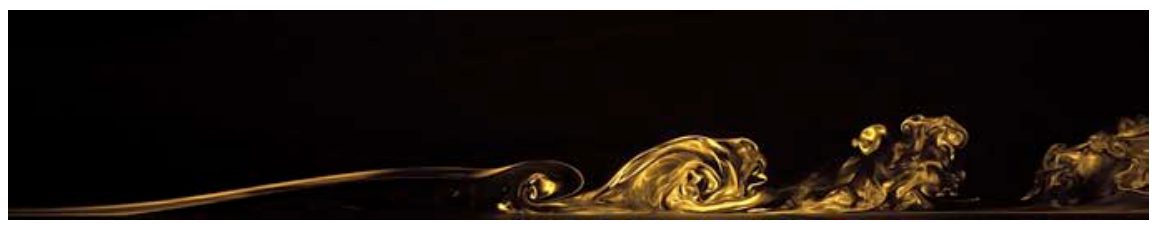

(b)

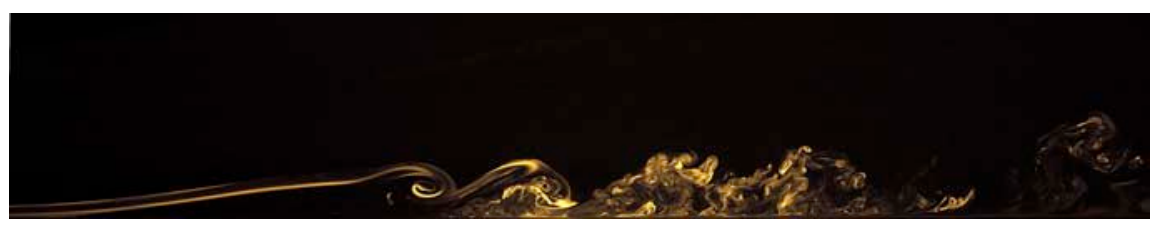

(c)

Figure 9. LIF images showing the instantaneous structure of the laminar separation bubble at three different upstream flow velocities. Freestream velocities were (a) $U_{\infty}=0.027 \mathrm{~m} / \mathrm{s} ;$ (b) $U_{\infty}=0.054 \mathrm{~m} / \mathrm{s} ;$ (c) $U_{\infty}=0.087 \mathrm{~m} / \mathrm{s}$.

The measured instantaneous flow field for one full period of the observed temporal shedding cycle is shown in figure 10 for an inflow velocity $U_{\infty}=0.087 \mathrm{~m} / \mathrm{s}$. The streamline patterns and the corresponding spanwise vorticity contours are shown at six equally spaced time intervals within the shedding cycle. The most obvious flow pattern is the periodic shedding of the small flow structure (a) containing reverse flow from the main recirculation region of the separation bubble. For every shedding cycle, the separation bubble seems to grow until the separation region splits into two separate cells. Subsequent to its shedding, this structure convects downstream at approximately half of the local freestream velocity, and eventually seems to breakdown. The dashed line in the vorticity contour follows one such shed vortex (a). The cycle repeats itself and the state of the separation bubble returns to the one seen at the start of the cycle. The upstream portion of the separation bubble is largely unaffected by the vortex shedding process. It is also apparent from the vorticity contours that the decay of the shed vortices is very rapid, which is also evident in the flow visualization pictures. Figure 11 (left) shows the time history of spanwise vorticity for an inflow velocity of $U_{\infty}=0.027 \mathrm{~m} / \mathrm{s}$. The time interval between each frame presented is 3.6 seconds. Even though the process of vortex shedding is the same as decribed in the preceding lines, it was observed that the shedding was not as regular. This is evident in the velocity time-series plot shown in figure 11 (right). The signal is strongly modulated. It is surmised here that the bubble may be on the verge of becoming a 'long' bubble. It is also worth mentioning that much longer time-series of data are required in order to obtain the true time-averaged topology of the separation bubble. The time-averaged bubble height appears to be lower than expected (see figure 8) at this velocity, possibly because of the aforementioned reason. From the observations made, it is surmised that the roll-up of the shear layer starts at a location prior to the maximum height of the bubble, with the actual shedding being observed at the location of maximum bubble height. The roll-up of the shear layer and its eventual breakdown is seen as being typically a result of Kelvin-Helmholtz instability (Bernal and Roshko ${ }^{20}$, Spalart and Strelets ${ }^{21}$, Fasel and Postl $1^{22}$, Hosseinverdi and $\mathrm{Fasel}^{23}$ ). 

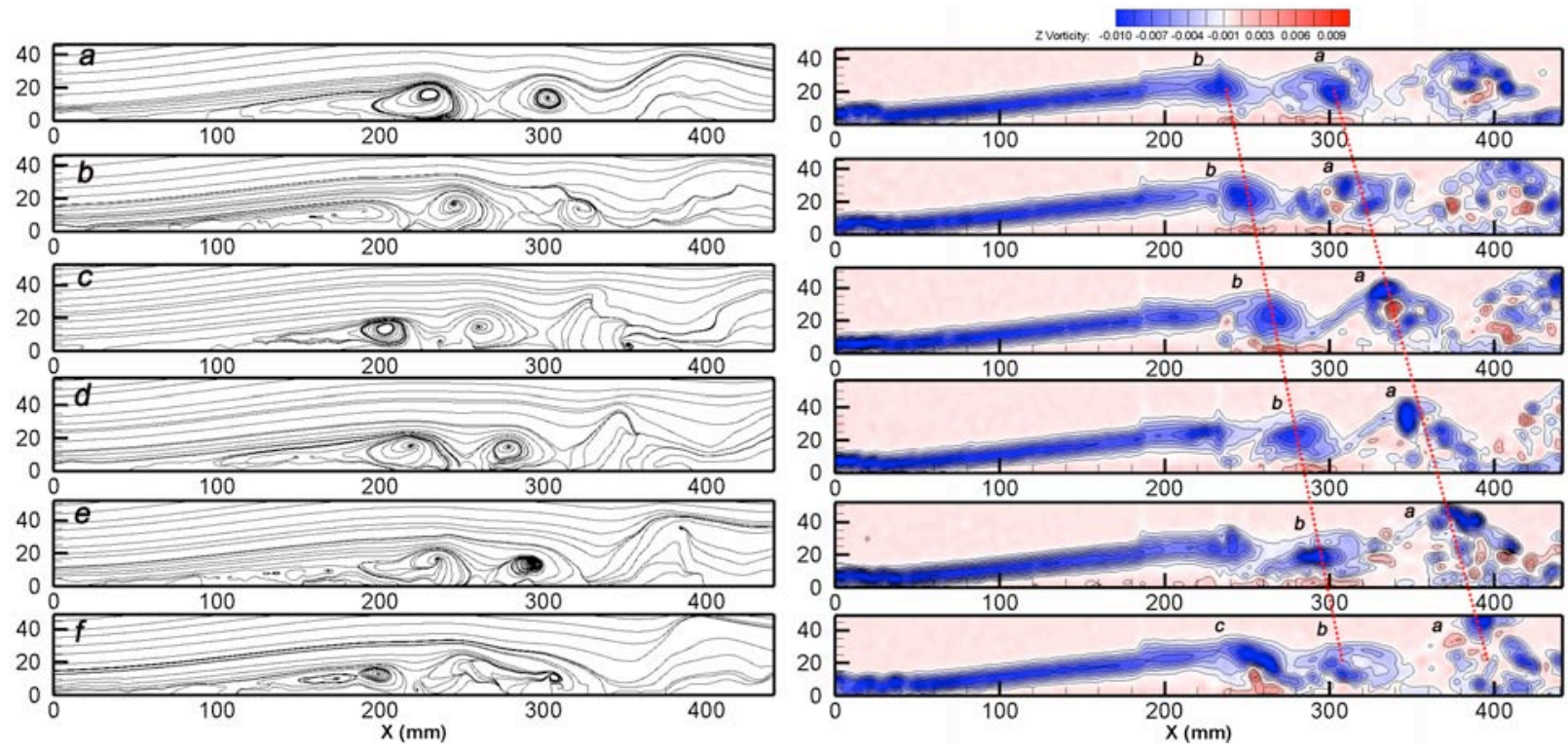

Figure 10. Streamlines (left) and spanwise vorticity contours (right) at six equal time intervals during one full cycle of the dominant frequency. $U_{\infty}=0.087 \mathrm{~m} / \mathrm{s}$. Time between frames $=0.8$ seconds.

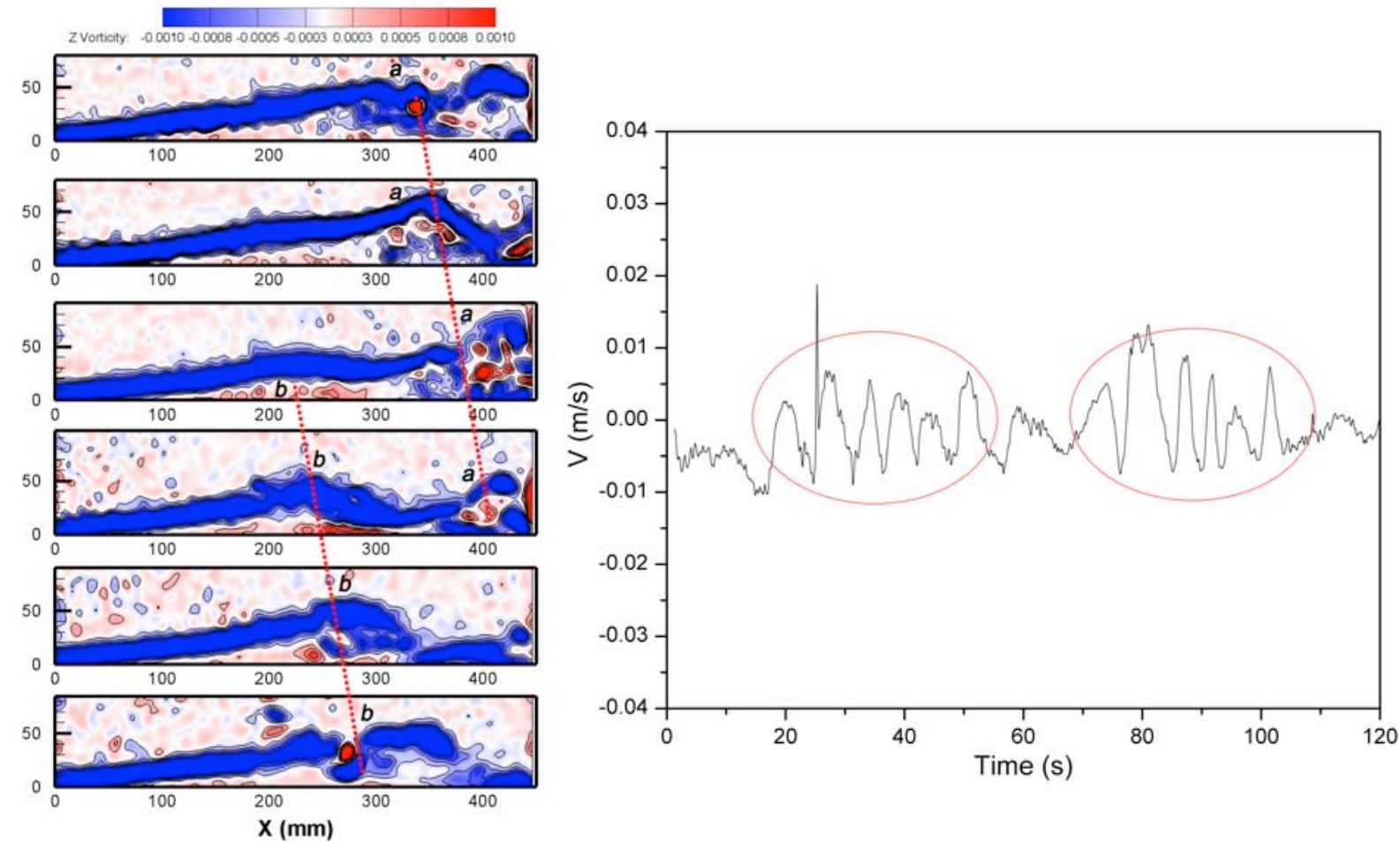

Figure 11. Spanwise vorticity contours (left) at six equal time intervals during one full cycle of the dominant frequency. $U_{\infty}=0.027 \mathrm{~m} / \mathrm{s}$. Time between frames $=3.6$ seconds. Time series of $v$ velocity variation, acquired at a location $x=200 \mathrm{~mm}$ and $y=45 \mathrm{~mm}$.

\section{Shedding frequency}

Time series data downstream of the separartion reattachment location were analysed to ascertain the frequency of the vortex shedding. From the previous section it is safe to assume that the natural shedding frequency of a laminar separartion bubble is strongly dependent on the inflow velocity and on the imposed pressure gradient. Fluctuations of the vertical component of velocity were Fourier transformed to determine the shedding frequency.

9

American Institute of Aeronautics and Astronautics 
Time series data extracted near the separation point do not show any periodicity. This is due to the fact that the upstream portion of the separation bubble is steady. Figure 12 shows the time series of vertical velocity for an inflow velocity of $U_{\infty}=0.038 \mathrm{~m} / \mathrm{s}$. The fluctuations seen in the time history indicate passing of large flow structures. The spectrum shows a dominant peak at a frequency of $0.25 \mathrm{~Hz}$. The broadband nature of the frequency spectrum is likely due to effects of the free-stream turbulence in the water tunnel.
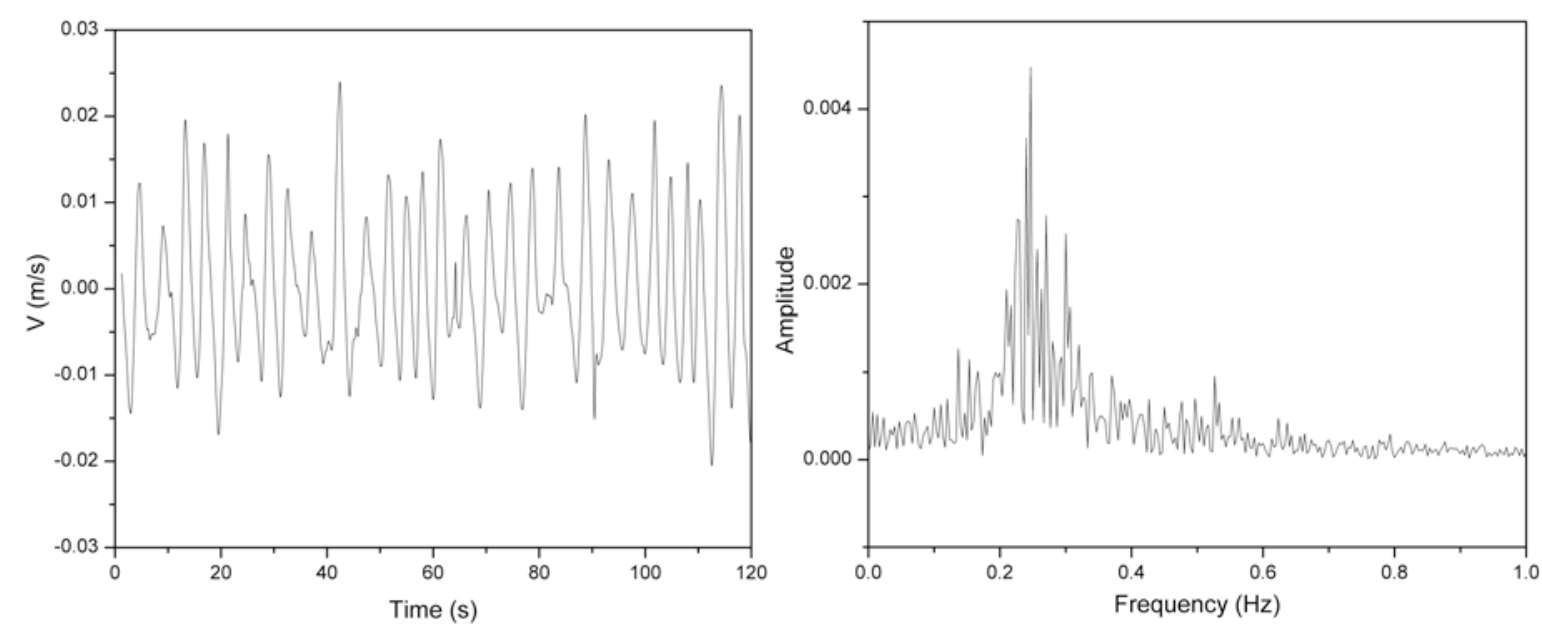

Figure 10. Velocity time history (left) and frequency spectrum (right) from Fourier analysis at an inflow velocity of $U_{\infty}=\mathbf{0 . 0 3 8 m} / \mathrm{s}$.

\begin{tabular}{|c|c|c|c|}
\hline$U_{\infty}(m / s)$ & $\begin{array}{c}\text { Bubble length } \\
l(\mathrm{~m})\end{array}$ & $\begin{array}{c}\text { Frequency of shedding, } \\
f(\mathrm{~Hz})\end{array}$ & $\begin{array}{c}\text { Strouhal Number } \\
S t=f l / U_{\infty}\end{array}$ \\
\hline 0.028 & 0.375 & 0.2 & 2.68 \\
0.038 & 0.38 & 0.25 & 2.50 \\
0.047 & 0.37 & 0.3 & 2.36 \\
0.059 & 0.35 & 0.4 & 2.37 \\
0.066 & 0.33 & 0.52 & 2.60 \\
0.074 & 0.32 & 0.55 & 2.38 \\
0.087 & 0.31 & 0.60 & 2.33 \\
\hline
\end{tabular}

Table 1. Summary of vortex shedding frequencies (dimensional and non-dimensional) and bubble lengths.

The shedding frequencies for several flow conditions (upstream velocity, pressure gradient) were determined and were non-dimensionalised using the bubble length and the free-stream velocity to determine a Strouhal number. In the present study it was found that the Stouhal number was more or less constant with stronger deviations being seen for case with the lowest freestream velocity. This indicates that the non-dimensionalized frequency is more or less independent of Reynolds number.

\section{Conclusion}

Separation of a laminar boundary layer under the influence of an adverse pressure gradient, which was induced by an inverted wing in the free stream, was studied. The effect of freestream velocity and the imposed pressure gradient was examined. For a given freestream velocity, the intensity of the pressure gradient was seen to have an influence on the bubble length and maximum height. Analysis of the unsteady flow in the separation bubbles was carried out. The dominant vortex shedding frequencies were obtained by Fourier analysis of the time series of the velocity signals in the shear layer. For the lowest flow velocity investigated, the bubble was observed to shed vortices only intermittently, with the time series of the velocity being strongly modulated. The vortex shedding for higher velocities was more regular, leading to a frequency spectrum with distinct peaks. The non-dimensionalised frequency using bubble length and freestream velocity was found to be fairly constant, except for the case with the lowest freestream velocity. 


\section{Acknowledgments}

The work presented was funded by the Air Force Office of Scientific Research (AFOSR) under grant number FA9550-05-1-0214 with Dr. Douglas Smith serving as program manager.

\section{References}

${ }^{1}$ McMichael, JM., \& Francis, MS., Micro-air-vehicles- toward a new dimension in flight. URL: http://www.darpa.mil/tto/MAV/ mav_auvsi.html, 1997.

${ }^{2}$ Mayle, R.E., "The role of laminar-turbulent transition in gas turbine engines", ASME Journal of Turbomachinery, Vol. 113 pp.509-37, 1991.

${ }^{3}$ Radi, A., \& Fasel, H., Experimental investigation of laminar separation bubbles on a flat plate, AIAA Conf., AIAA 20104482-292, 2010.

${ }^{4}$ Gaster, M., "The Structure and behaviour of laminar separation bubbles", Aeronautical Research Council Reports and Memoranda, pp. 1-32, 1967.

${ }^{5}$ Merzkirch, W., Flow Visualization. Academic Press, New York, second edition, 1987.

${ }^{6} \mathrm{LaVision} \mathrm{GmbH}$, Tools for DaVis - DaVis flowmaster software. DaVis Flowmaster Software Manual for DaVis 7.0 (2004).

${ }^{7}$ Horton, H.P., "Laminar separation bubbles in two and three dimensional incompressible flow", Ph.D Thesis, University of London. 1968.

${ }^{8}$ Häggmark, C.P., Hildings, C., \& Henningson, D.S., “A numerical and experimental study of a transitional separation bubble", Aerospace Science and Technology, Vol 5, Issue 5, pp 317-328, 2001.

${ }^{9}$ Gad-el-Hak, M., Flow control: passive, active, and reactive flow management, Cambridge University Press, Cambridge, 2000.

${ }^{10}$ Jones, B.M., "Stalling”, Journal of the Royal Aeronautical Society, Vol 38, pp. 747-770, 1938.

${ }^{11}$ Owen, P.R., \& Klanfer, L., "On the laminar boundary layer separation from the leading edge of a thin aerofoil", A.R.C Technical Report, Conference Proceedings 220, 1953.

${ }^{12}$ Van den Berg, B., "Reynolds number and Mach number effects on the maximum lift and stalling characteristics of wings at low speeds" NLR Technical Report 69025U, 1969.

${ }^{13}$ Diwan, S.S., Chetan, S.J. \& Ramesh, O.N., “On the bursting criterion for laminar separation bubbles". In Sixth IUTAM Symposium on Laminar-Turbulent Transition (ed. R. Govindarajan), Fluid Mechanics and Its Applications, Vol. 78, pp. 401407. Springer. 2006.

${ }^{14}$ Marxen, O \& Henningson, D.S., "The effect of small-amplitude convective disturbances on the size and bursting of a laminar separation bubble”. Journal of Fluid Mechanics, Vol. 671, pp. 1-33, 2011

${ }^{15}$ Pauley., L.L., Moin., P. \& Reynolds., W.C. "The structure of two-dimensional separation". Journal of Fluid Mechanics. Vol. 220, pp 397-411. 1990

${ }^{16}$ Ripley., M.D. \& Pauley., L.L. "The unsteady structure of two-dimensional separation". Physics of Fluids A. Vol.5, pp 30993106. 1993.

${ }^{17}$ Lin., J.C.M. \& Pauley., L.L. "Low-Reynolds-number separation on an aerofoil". AIAA Journal. Vol 34, pp 1570-1577. 1996.

${ }^{18}$ Alam., M. \& Sandham, N.D. "Direct numerical simulation of 'short' laminar separation bubbles with turbulent reattachment". Journal of Fluid Mechanics. Vol 410, pp 1-28. 2000.

${ }^{19}$ Hammond, D.A. \& Redekopp, L.G. 1998 "Local and global instability properties of separation bubbles". European. Journal of Mechanics. B/Fluids. Vol 17. pp 145-164. 1998.

${ }^{20}$ Bernal, L.P. \& Roshko., A. "Streamwise vortex structure in plane mixing layers," Journal of Fluid Mechanics., Vol 170, pp. 499-525.1986.

${ }^{21}$ Spalart, P.R. \& Strelets., M.K. "Mechanisms of transition and heat transfer in a separation bubble". Journal of Fluid Mechanics. Vol 403. pp. 329-349. 2000.

${ }^{22}$ Fasel, H.F. \& Postl, D., "Interaction of separation and transition in boundary layers: Direct Numerical Simulations". In Sixth IUTAM Symposium on Laminar-Turbulent Transition (ed. R. Govindarajan), Fluid Mechanics and Its Applications, Vol. 78, pp. 71-88. Springer. 2006.

${ }^{23}$ Hosseinverdi, S., Fasel, H. F., "Investigation of Transition and Separation in the Presence of Free-Stream Turbulence using Direct Numerical Simulations,” AIAA paper, AIAA 2012-96, 2012. 